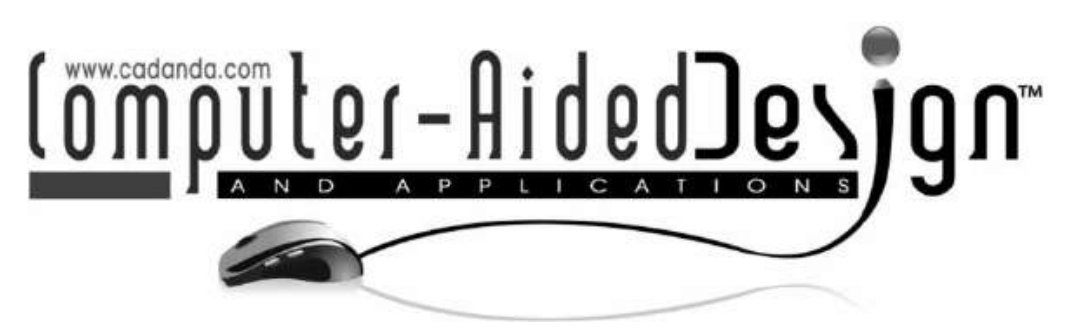

\title{
Topological Validation of Midsurface Computed from Sheet Metal Part
}

\author{
Yogesh H Kulkarni ${ }^{1}$, Anil Sahasrabudhe ${ }^{2}$ and Mukund Kale ${ }^{3}$ \\ ${ }^{1}$ College of Engineering Pune, India, kulkarniyh12.mech@coep.ac.in \\ ${ }^{2}$ College of Engineering Pune, India, director@coep.ac.in \\ ${ }^{3}$ Siemens PLM, Pune, India, Mukund_kale@hotmail.com
}

\begin{abstract}
During the initial stages of iterative design process, a quick CAE (Computer-aided Engineering) analysis of the CAD (Computer-aided Design) models is needed. To reduce the computational resources and time needed for such analysis, the models are often simplified by removing the irrelevant details and are abstracted by reducing the dimension, wherever appropriate. Thin-walled parts, such as sheet metal parts are often abstracted to a set of surfaces lying midway, called mid-surface. The mid-surface is expected to mimic the shape of the original solid, both geometrically and topologically. Widely-used methods of accessing the quality of the mid-surface are geometric. Hausdorff distance from the midsurface its original solid is computed to find the gaps and medial-ness. Accuracy of such methods depends on the sampling as well as on the complexity of the surface representation, making them computationally intensive and error-prone.

This paper provides a topological method for verification, which is computationally simple and robust. A novel topological transformation relationship has been derived between a sheet metal part (solid) to its mid-surface (surface), in both directions (solid-to-surface and surface-to-solid) which can be used to compare the predicted vs actual entities. Simple as well as practical shapes have been tested to prove the efficacy of the newly-derived formulation.
\end{abstract}

Keywords: CAD, CAE, topology, Euler characteristics, Betti numbers, sheet metal parts, mid-surface, cellular decomposition

\section{INTRODUCTION}

Mid-surface is an abstracted representation of a thinwalled solid, used mainly for creating shell elements in the CAE meshing process. It can also be used as a shape-signature in shape matching/retrieval. It is expected to express the contiguous flow of the solid's shape [15]. So, to be truly effective, the mid-surface needs to mimic the original solid, in both, geometrical and topological sense. Geometrically, the shape of the mid-surface should be such that it lies in the middle (at half the thickness) of the solid. Topologically, the connectivity between the mid-surface patches should be similar to that of their corresponding sub-shapes in the original solid.

There are a large number of methods to compute the mid-surface. They work on different input types of the original solid, such as, faceted mesh, Boundary Representation (Brep) solids, feature-based CAD models, etc. Quality of the output mid-surface depends on the shape-characteristics of the original thin-walled solid.

\subsection{Thin-walled Solids}

Many thin-walled solids are from the sheet metal domain. These are unique in both, geometrical and topological sense. They are characterized by:

- Constant thickness: Sheet metal parts are made up of constant thickness blank roll.

- Absences: There are no blind holes but only through holes, if any.

- Degeneracy: There are no degenerate capping thickness faces (like "Wedge").

- Cavities: There are no embedded volumes or cavities ("bubbles").

Quality of the output mid-surface depends on the complexity of the original solid. The topological validation method for the mid-surface developed in this work has been devised for solids exhibiting sheet metal shape characteristics. Such thin-walled solids, with constant thickness, pose lesser problems in the computation of the mid-surface as well as in 
the validation of the mid-surface, than the ones with variable thicknesses. Although the validation method mentioned in this work is derived for constant thickness solids, it can be extended to thin-walled parts with variable thickness as well, e.g., injection-molded plastic parts having drafts. Shape with or without draft angle are topologically the same, so the formulation developed in this work applies equivalently.

Many of the commercial sheet metal CAD modelers represent the thin-walled shape using a data-structure called Boundary Representation (Brep). Section 2 provides the characteristics of Brep and its classification into manifold and non-manifold representations. In this work, the term 'manifold' refers to an object which is bound, closed and homeomorphic to a topological sphere (also known as 2-manifold), whereas 'non-manifold' object does not have such restrictions of closure and completeness. This work uses 'non-manifold' mainly for surfaces, unless stated otherwise.

\subsection{Mid-surface Computation}

Mid-surface computation has been a widelyresearched topic and there are many methods such as Medial Axis Transform, Chordal Axis Transform, and Mid-surface Abstraction, etc. [18]. Out of these, very few are based on the explicit shape transformation operators.

Sheen et al. [17] used the deflation process to compute mid-surface from a solid. Their algorithm did not change the topology but just reduced the capping entities to zero size. The problem with such an approach could be that the degenerated topology would be potentially detrimental to the downstream modeling operations.

Lee [10] proposed topological operators to transform a sheet of solid topology into a thin-walled solid by face geometry replacement. However, this method could pose difficulty in representing the adjacency relationships.

Even after extensive research in the academic domain and wide availability in commercial implementations, mid-surface quality is still a concern. It suffers from errors like gaps, overlaps, missing surfaces, etc. Validating the output mid-surface is a critical step in assessing the quality, after which corrective actions can be taken.

\subsection{Mid-surface Validation}

To verify the quality of the mid-surface, the following methods are used:

- Manual: Manual inspection for errors such as missing surfaces, connection gaps, overlaps, etc. One needs to ensure that the mid-surface lies midway and is continuous throughout, especially at the connections and steps. This method is obviously tedious, time-consuming and errorprone.

- Inspection Tools: Tools provided in the CADCAE packages can detect gaps and overlaps but they cannot detect the correctness of the mid-surface at critical locations, such as connections and steps, where expectations could be subjective.

- Geometric Tools: Hausdorff distance from the mid-surface to its original solid is computed. Accuracy of such methods depends on the sampling as well as on the complexity of the surface representation, making them computationally intensive and error-prone.

- Topological Validation: This involves comparison of a number of predicated topological entities with the actual ones, and if there is a mismatch, the problem is detected. Here, the geometry of the shape is ignored. It has an advantage over geometric validation since computationally-intensive distance calculations are not performed.

This work proposes a novel method for the topological validation of mid-surface of thin-walled solids.

\subsection{Topological Validation}

Topological validation proposed in this work proposes two transformations with which the quality of a mid-surface can be assessed. First, solid-to-surface, where, the dimension-reduction transformation equations are applied to the thin-walled solid to predict the topological entities of the corresponding midsurface and then compared with the actual topological entities of the output mid-surface. In the second approach, surface-to-solid, dimension-addition transformation equations are applied to the output mid-surface to predict the topological entities of the corresponding thin-walled solid, and then compared with the actual topological entities of the thin-walled solid.

The validation method presented in this work cannot be used in isolation from geometry. As the midsurface is applicable only for the thin-walled solids, it is not computed and validated using this method for thick solids. But such differentiation of the solidshape, being thick or thin, is 'geometrical' and not 'topological', which this method itself cannot detect. So, the work presented below should be used only for the known thin-walled solids for which mid-surface are computable.

Use of topology for assessing the quality of midsurface is not widespread and there are very few such attempts reported in the literature.

Lipson [13] stated that a topological invariant for all the sheet metal parts and thin-walled objects can be used as a necessary condition for topological validity and reasoning. 
Lockett and Guenov [14] used both geometric and topological variants for checking the validity of the mid-surface. For geometric validation, they used the Hausdorff distance between a mid-surface and its corresponding principal faces (pairs). For topological validation, they used proximity groups adjusted by an angle criterion. The main limitation of their approach appears that the geometric criteria (closest distance proximity or angle between faces) are used in the topological validations, which, ideally, should not be the case.

Apart from CAE, skeletal structures such as midsurface are used in CAD model comparison solutions such as shape-based retrieval, similarity assessment and difference identification [3]. Skeletal graph matching is one of the prominent techniques [7] used for similarity assessment. A topologically-valid midsurface represents sub-shape connectivity better and thus acts as more effective shape-signature in the model comparison.

The work presented here, provides with transformation relations and a topological variant, based purely on the combinatorial topology for determining the validity of a midsurface computed from a sheet metal part.

\section{PRELIMINARIES}

\subsection{Boundary Representation (Brep)}

Brep is composed of two parts: topology and geometry. Topological elements are shells, faces, edges, vertices, etc. They are mentioned in the descending order of the topological dimensionality:

- shell (s) is a connected set of faces.

- face $(f)$ is a bounded portion of a surface (geometry).

- loop (l) is a circuit of edges bounding a face.

- half-edges (he) are used to create a loop.

- edge (e) is a bounded portion of a curve (geometry).

- $\operatorname{vertex}(v)$ lies at a point (geometry).

Validity of the Brep model is checked using the Euler-Poincaré equation.

\subsubsection{Euler-Poincaré equation}

Euler's equation for polyhedral solids is:

$$
v-e+f=2
$$

where, $v, e$, and $f$ represent the number of vertices, edges and faces respectively. It was discovered by Leonhard Euler in 1752 and was later generalized by Lhuilier [9] as follows:

$$
v-e+f=2-2 g
$$

where, $g$ represents genus, the number of holes $h$ or handles $(g$ and $h$ are considered interchangeable in this paper). Later on, Schläffi and Poincaré also generalized the formula to the higher dimensional n-polytopes.

The Euler characteristic $(\chi)$ for combinatorial cell complexes or polyhedral solids is defined as follows:

$$
\sum_{i=0}^{D}(-1)^{i} N_{i}=\sum_{i=0}^{D}(-1)^{i} \beta_{i}=\chi
$$

For dimensions up to $3(i=3)$, equation (1) simplifies to

$$
N_{o}-N_{1}+N_{2}=\beta_{0}-\beta_{1}+\beta_{2}
$$

where, Ns are topological entities of the dimension 0,1 and 2 respectively and $\beta s$ are Betti Numbers. $\beta_{0}$, $\beta_{1}$ and $\beta_{2}$ correspond to the number of connected components, holes and cavities, respectively [16].

Two homeomorphic topological spaces will have the same Euler characteristic and Betti numbers.

Most of the CAD models are made up of solids (manifolds), since they are considered to be complete and having their own volumes. A manifold-solid model can only represent one closed volume minus its internal structure. It cannot represent heterogeneous possibilities such as wires (curves), sheets (surfaces), and solids (volumes) together, which although, are not possible in the real world, but are possible during the intermediate stages of design [26]. A non-manifold model is a generic modeling framework which encompasses all these items in a single framework [12].

The Euler characteristic $(\chi)$, in terms of Betti numbers, provides a generic invariant for a shape of any dimension. Manifestation of the Betti numbers in different dimensions is different. So, when a thin-walled solid is transformed into its corresponding mid-surface, the interpretation of the Betti numbers changes from the manifold domain to the non-manifold domain.

\subsubsection{Manifold-solids}

The Euler Poincaré equation for manifold-solids is:

$$
v-e+(f-r)=2(s-h)
$$

Its equivalence with equation (2) is as follows:

- $N_{0}=v:$ number of vertices

- $N_{1}=e$ : number of edges

- $N_{2}=(f-r)$ : number of faces $(f)$ - additional artifact edges corresponding to inner loops $(r)$

- $\beta_{0}=s$ : number of components or disjoint parts (shells)

- $\beta_{1}=2 h$ : number of independent closed curves drawn without splitting. Twice the genus $g$ or $h$. For Torus, there are two such circles and one genus-hole $(2 \mathrm{~h})$.

- $\beta_{2}=s$ : number of space regions created by connected surfaces. For an open surface $\beta_{0}=1$ and 
$\beta_{2}=0$ whereas for closed surface, $\beta_{2}$ is equal to $\beta_{0}$ which is equal to $s$.

\subsubsection{Non-manifold-surfaces}

Solids found in the real world have the property that on any point on the boundary, a small enough sphere at that location is split into two pieces, one inside and one outside the object. Non-manifolds do not obey this rule [9]. Weiler ([20]) can be attributed for the first significant contribution in defining the nonmanifold data structure, called Radial Edge Structure. Core to this data structure lies the radial cycle, which is an ordered list of faces around an edge. Similar to manifold, the equation for non-manifold topology is: [25]

$$
v-e+(f-r)=s-h
$$

Its equivalence with equation (2) is as follows:

- $N_{0}=v$ : number of vertices

- $N_{1}=e$ : number of edges

- $N_{2}=(f-r)$ : number of faces $(f)$ - inner loops $(r)$

- $\beta_{0}=s$ : number of components or disjoint parts (shells)

- $\beta_{1}=h$ : number of independent closed curves drawn without splitting. Inner holes ( $g$ or $h$ ).

- $\beta_{2}=0$ : number of space regions created by connected surfaces is not present; so it is 0 .

\subsection{Cellular Decomposition}

Cellular topology is one of the prominent representations in solid modeling. The fundamental unit is called Cell, which has dimensionality $0 / 1 / 2 / 3$ and can have adjacency to its neighbor denoted

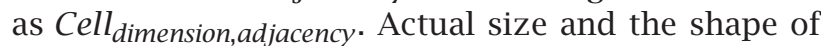
the cells can vary based on the underlying geometry (Table 1).

\subsubsection{Cellular entities}

Cellular Decomposition is a process by which a shape (called "Original solid") is split in to multiple subshapes (called "Cells"). According to Chen et al. [5], a cellular model includes the topologies of various dimensions.

$$
M=\left(\cup_{i=1}^{q} C_{i}^{0}\right) \cup\left(\cup_{j=1}^{r} C_{j}^{0}\right) \cup\left(\cup_{k=1}^{S} C_{k}^{0}\right) \cup\left(\cup_{l=1}^{t} C_{l}^{0}\right)
$$

where, $C_{i}^{0}$ are 0 -dimensional vertices, $C_{j}^{1}$ are 1dimensional edges, $C_{k}^{2}$ are 2-dimensional faces, and $C_{i}^{3}$ are 3-dimensional solids.

The cells have the following properties:

- Boundary: Except $C_{i}^{0}$ cells, all cells are bound by cells with a dimension lower by 1 .

- Overlap: No cells overlap. $C_{i}^{a} \cap C_{i}^{b}=\emptyset(0 \leq a$; $b \leq 3 ;(a=b)(i \neq j))$

- Nature: Either additive or subtractive.

\begin{tabular}{|c|c|c|}
\hline $\mathrm{Cell}_{3, *}$ & $\begin{array}{l}\text { 3D cells (solids), } \\
\text { topologically } \\
\text { similar to a simple } \\
\text { plate }\end{array}$ & $\begin{array}{l}\text { faces }=6 \\
\text { edges }=12 \\
\text { vertices }=8\end{array}$ \\
\hline $\mathrm{Cell}_{2, *}$ & $\begin{array}{l}\text { 2D cells, topologically } \\
\text { similar to a planar } \\
\text { surface }\end{array}$ & $\begin{array}{l}\text { faces }=1 \\
\text { edges }=4 \\
\text { vertices }=4\end{array}$ \\
\hline $\operatorname{Cell}_{1, *}$ & $\begin{array}{l}\text { 1D cells, topologically } \\
\text { equivalent to a line }\end{array}$ & $\begin{array}{l}\text { edges }=1 \\
\text { vertices }=2\end{array}$ \\
\hline $\mathrm{Cell}_{3, h} \circlearrowright$ & $\begin{array}{l}\text { Hole is assumed } \\
\text { to be cylindrical } \\
\text { throughout (true } \\
\text { for sheet metal } \\
\text { parts) }\end{array}$ & $\begin{array}{l}\text { edges }=1 \\
\text { vertices }=1\end{array}$ \\
\hline
\end{tabular}

Cells can be of the following types:

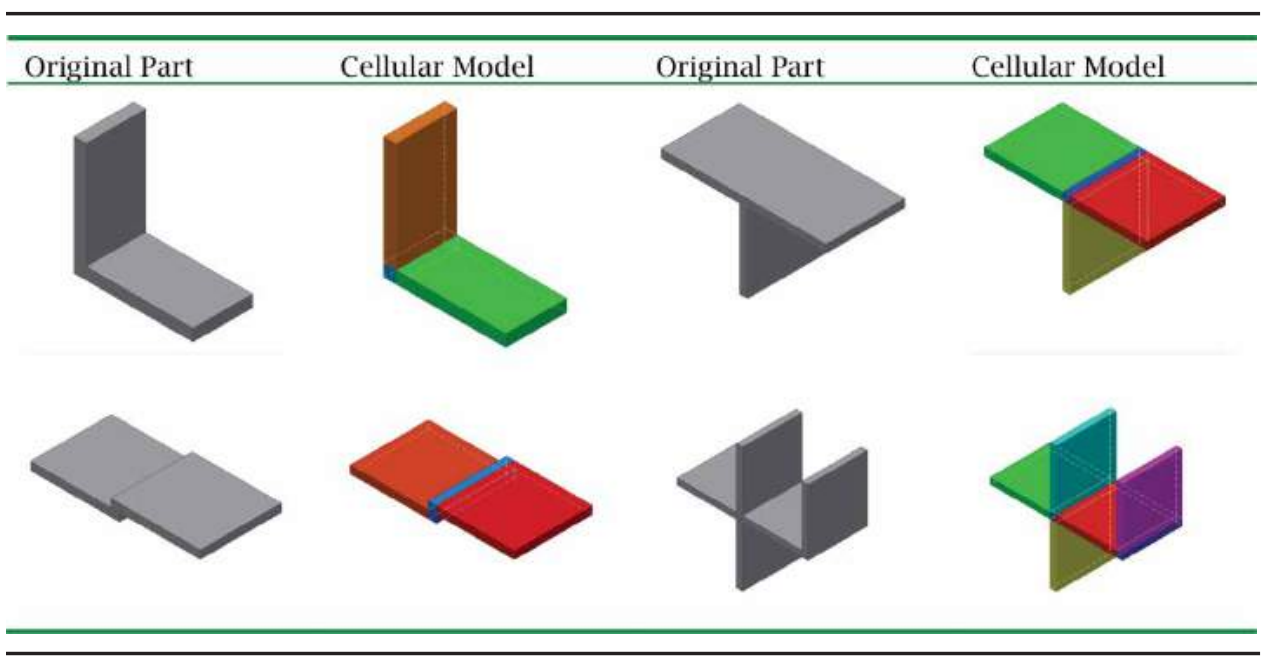

Tab. 1: Decomposition of shapes into cells. 


\subsubsection{Classification of cells}

When the original solid is decomposed into distinguishable cells, new interface boundaries are introduced. Newly-created intersecting volumes or touching boundaries are called interface cells, which can be either 3D (solids) or 2D (faces) respectively.

Two shapes touching each other with an area overlap, is called 2D-interface

Two shapes touching each other with a volume overlap, is called 3D-interface

If two bodies spatially overlap then they are split to form the 3D-interface (Fig. 2) cell. In case of 2Dinterface (Fig. 1), adjoining faces of the overlapping face are extended [23] and used as a cutting tool to create the intersecting volume, the 3D-interface (Fig. 2) cell.

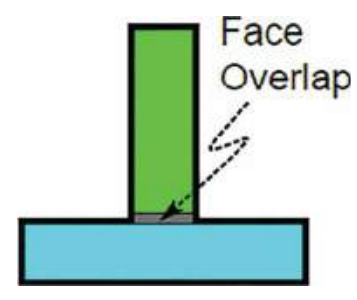

Fig. 1: 2D-interface.

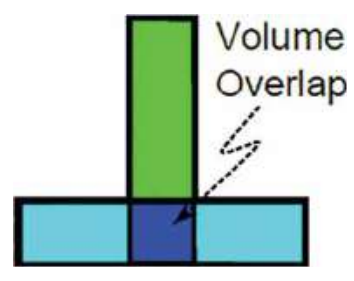

Fig. 2: 3D-interface.

Prefix $s$ is applied if the Cell is from the original solid, $i$ if it is of a newly introduced interface type, (Fig. 3 ) and $m$ for midsurface cells.

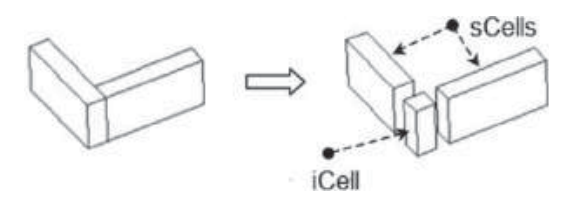

Fig. 3: Decomposition and classification [19].

\section{TOPOLOGICAL VALIDATION OF MIDSURFACE}

Topological validation can be performed using two methods:

- Solid-to-Surface: Find the relationship between the topological entities of a thin-walled solid and its corresponding midsurface. Check whether the predicted midsurface entities validate the non-manifold equation (4).

- Surface-to-Solid: Predict the topological entities of a possible thin-walled solid that could be generated by thickening of the given midsurface. These predicted entities can be validated against the entities of the original thin-walled solid as well as with the manifold equation (3).

Following are the ways in which some of the midsurface errors can be detected using the predicted entities:

- Missing Surfaces: Missing surfaces result in lesser number of edges and vertices

- Missing Connections: Gaps result in lesser radial edges and vertices

\subsection{Sheet Metal Solid to Midsurface Transformation}

Given a thin-walled sheet metal solid, an approach is given below to propose the dimension-reductiontransformation equations for predicting the topological entities of its corresponding midsurface. The given original solid is first decomposed into sub-volumes, called as "Cells" (Table 1) and then the dimensionreduction-transformation is derived.

Many commercial as well as academic methods are available for cellular-volumetric decomposition [23, 6, 4, 1, and 9]. Woo (maximal cells [21]), Boussuge (Extrusion decomposition [2]), Wu (Sweep Decomposition [24]), Woo (Protrusion decomposition [22]), etc. are some of the known cellular decomposition methods used for feature recognition (FR).

Cellular decomposition starts facing problems as the complexity of the original solid increases. The method becomes very slow as the number of cells increases [21]. If the cutting faces are extended infinitely and intersected with the whole solid then they generate a large number of unnecessary cells. If the splits are not clean, it may generate degenerate entities such as edges and vertices. The topological validation method presented here assumes 'clean' cellular decomposition and if it is not so, then the validation results could be unpredictable.

\subsubsection{Steps: Topological dimension reduction}

Topological transformation of a solid (3D cells) to its corresponding midsurface (2D cells) is as follows:

- $\operatorname{sCell}_{3, n}$ : Solid cell with $n$ touching sides transforms into a midsurface cell $m \mathrm{Cell}_{2, n}$, a surface having $n$ empty edges. Its topological entities are denoted as

$$
f=1 ; e=4-n ; v=4-2 n
$$




\begin{tabular}{|c|c|c|c|c|}
\hline Solid & Midsurface & sCells & mCells & Predicted Midsurface Entities \\
\hline & & $s_{C e l l}^{3,0}$ & $m C e l l_{2,0}$ & $1 f+(4-0) e+(4-2 \times 0) v=1 f+4 e+4 v$ \\
\hline & & $\begin{array}{l}2 \times \mathrm{sCell}_{3,1}+ \\
\text { iCell }_{3,2}\end{array}$ & $\begin{array}{l}2 \times \mathrm{mCell}_{2,1}+ \\
\mathrm{mCell}_{1,2}\end{array}$ & $\begin{array}{l}2 \times(1 f+(4-1) e+(4-2 \times 1) v)+ \\
\quad(1 e+2 v)=2 f+7 e+6 v\end{array}$ \\
\hline & & $\begin{array}{l}3 \times \mathrm{sCell}_{3,1}+ \\
\quad \mathrm{iCell}_{3,3}\end{array}$ & $\begin{array}{l}3 \times \mathrm{mCell}_{2,1}+ \\
\quad \mathrm{mCell}_{1,3}\end{array}$ & $\begin{array}{l}3 \times(1 f+(4-1) e+(4-2 \times 1) v)+ \\
\quad(1 e+2 v)=3 f+10 e+8 v\end{array}$ \\
\hline & & $\begin{array}{l}3 \times \text { sCell }_{3,1}+ \\
\quad \text { iCell }_{3,3}+\text { sCell }_{3, h}\end{array}$ & $\begin{array}{l}3 \times \mathrm{mCell}_{2,1}+ \\
\mathrm{mCell}_{1,3}+ \\
\\
\mathrm{mCell}_{2, h}\end{array}$ & $\begin{array}{l}3 \times(1 f+(4-1) e+(4-2 \times 1) v)+ \\
\quad(1 e+2 v)+(1 e+1 v)=3 f+11 e+9 v\end{array}$ \\
\hline & & $\begin{array}{l}2 \times \operatorname{sCell}_{3,1}+2 \times \\
\quad \mathrm{iCell}_{2,2}+\text { sCell }_{3,2}\end{array}$ & $\begin{array}{l}2 \times \mathrm{mCell}_{2,1}+ \\
2 \times \mathrm{mCell}_{1,2}+ \\
\quad m \mathrm{mell}_{2,2}\end{array}$ & $\begin{array}{l}2 \times(1 f+(4-1) e+(4-2 \times 1) v)+ \\
\quad 2 \times(1 e+2 v)+ \\
\quad(1 f+(4-2) e+(4-2 \times 2) v)= \\
\quad 3 f+10 e+8 v\end{array}$ \\
\hline
\end{tabular}

Tab. 2: Dimension-reduction-transformations.

- $s \mathrm{Cell}_{3, h}$ : Negative solid cell representing a through hole transforms into a midsurface cell $\mathrm{mCell}_{2, h}$, a hole in the surface. Its topological entities are denoted as

$$
e=1 ; v=1
$$

- iCell $_{3, n}$ : Interface solid cell with $n$ adjacent touching sides transforms into a midsurface cell $m \mathrm{Cell}_{2, h}$, a radial edge with $n$ leaves. Its topological entities are denoted as

$$
e=1 ; v=2
$$

- iCell $_{2,2}$ : Interface face cell touched from both sides transforms into a midsurface cell $m$ Cell $_{1,2}$, a radial edge with 2 leaves. Its topological entities are denoted as

$$
e=1 ; v=2
$$

\subsubsection{Examples}

Table 2 lists the various basic shapes and their dimension-reduction-transformations into the corresponding midsurfaces. It is evident that the predicted midsurface entities of these simple shapes match with the actual ones, thus the derived formulation works for these simple shapes.

Following is the verification for a relativelycomplex practical shape.

- Solid cells: $5 \times s$ Cell $_{3, h}+3 \times$ sCell $_{3,1}+13$ $\times \mathrm{sCell}_{3,2}+14 \times$ iCell $_{2,2}$
- Transformed midsurface cells: $5 \times \mathrm{mCell}_{2, h}+$ $3 \times$ mCell $_{2,1}+13 \times$ mCell $_{2,2}+14$ $\times$ mCell $_{1,2}$

- Predicted midsurface entities: $5(1 e+1 v)+$ $3(1 f+3 e+2 v)+13(1 f+2 e+0 v)$ $+14(1 e+2 v)=16 f+54 e+39 v$

The derived formulation (Eqn 7, 8, 5, 6) predicts correct topological entities for the midsurface. These, when substituted in the non-manifold equation (Eqn 4) also prove to be valid. With $s=1 ; r=5 ; h=5$, the equation matches both sides: $39-54+(16-5)=$ $1(1-5)$

\subsection{Midsurface to Sheet Metal Solid Transformation}

In this approach, given a midsurface, topological entities of its corresponding sheet metal solid are predicted. These predicted entities are verified to check whether they validate manifold equation (Eqn 3). Topological entities of a midsurface contain far richer (classifiable) topological information than its corresponding solid model. For example, midsurface of "T" shaped solid, which can be represented as Figure 4 has the following classified entities:

- Faces $(f)$ : Bound by two face-uses $f_{u}$

- Sharp Vertex $\left(v_{s}\right)$ : Connected to two edges of the same face

- Sharp Edge $\left(e_{s}\right)$ : Connected to two sharp vertices 


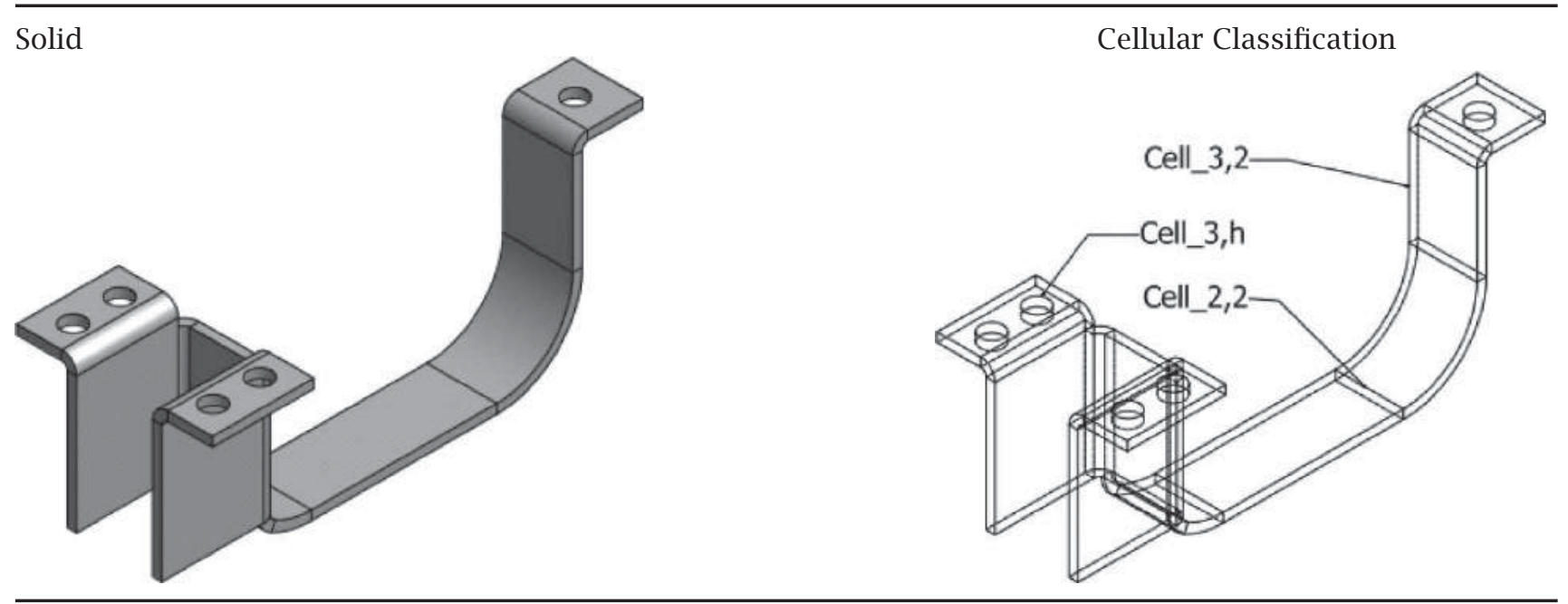

- Radial Vertex $\left(v_{r}\right)$ : Connected edges of different faces

- Degree $\left(n_{r}\right)$ at the radial edge is the number of faces attached to it

- Cross Radial Edge $\left(e_{r}\right)$ : Connected between two radial vertices and connects two different faces

- Side Radial Edge $\left(e_{r r}\right)$ : Connected between two radial vertices and is of same face

- Sharp Radial Edge $\left(e_{s r}\right)$ : Between sharp and radial vertex

- Internal Edge $\left(e_{i}\right)$ : Part of the inner loop

- Internal Vertex $\left(v_{i}\right)$ :Connected to the internal edge

- Internal Loop $\left(r_{i}\right)$ : Characterized by internal edges and vertices

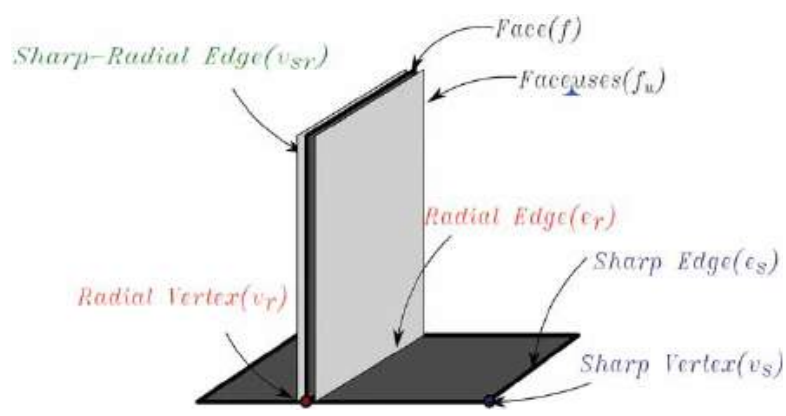

Fig. 4: Non-Manifold topological entities.

\subsubsection{Steps: Topological dimension addition}

Sheet Metal solid can be imagined to be the thickened midsurface [11]. The topological entities of the generated solid are calculated as per the following steps:

- Face-uses become the principal faces.

- Sharp vertices create the capping edges.
- Apart from edge-use loop corresponding to faceuse, a new loop is proposed for the side-capping faces. The loop is formed between two sharp vertices $\left(v_{s}\right)$ using more than one sharp $\left(e_{s r}\right)$ or side radial $\left(e_{r r}\right)$ edges but not using the cross radial edge $\left(e_{r}\right)$. Such independent paths creating individual side faces are called $\left(l_{p}\right)$.

- Loop between two sharp vertices. This gives rise to a singular capping face (Fig. 5a).

- Loop between three branched sharp vertices. This gives rise to a combined capping face (Fig. 5b).

- Loop between two sharp vertices with multiple radial vertices in between them. This gives rise to a combined capping face (Fig. 5c).

Topological entities in the thickened solid are predicted as follows:

- Manifold-Vertices $\left(v_{m}\right)=$ Double the sharp and internal vertices (one up and one below) + vertices for junctions of which are denoted by the summation of number of the radial vertices times their corresponding degrees.

$$
v_{m}=2\left(v_{s}+v_{i}\right)+\sum n_{r} v_{r}
$$

- Manifold-Edges $\left(\boldsymbol{e}_{\boldsymbol{m}}\right)=$ Double the sharp, sharpradial and internal edges (oset up and down) + degree times radial edges for offsets at junctions + sharp vertices for vertical-capping edges + internal vertices for vertical seam edges.

$$
e_{m}=2\left(e_{s}+e_{s r}++e_{r r}+e_{r}\right)+\sum n_{r} e_{r}+v_{s}+v_{i}
$$

- Manifold-Faces $\left(f_{m}\right)=$ Double the faces (offset up and down) + sharp edges for capping faces + paths to have one combined face + internal 


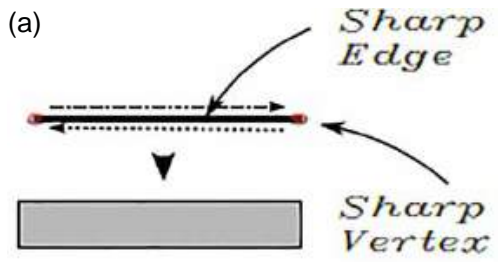

(b)

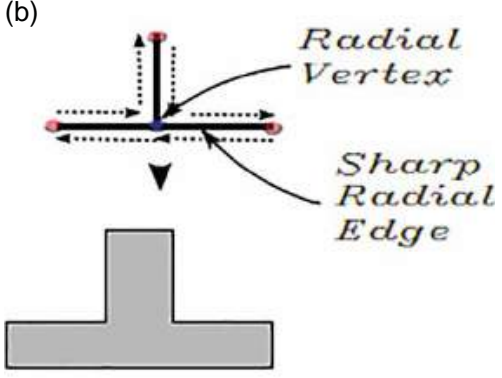

(c)
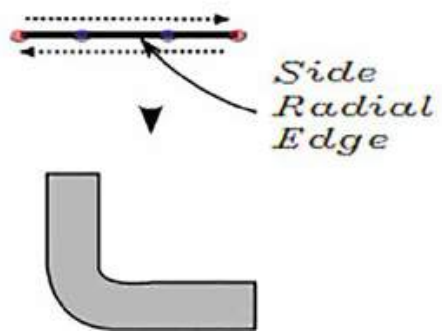

Fig. 5: Loops to faces. edges for capping internal faces.

$$
f_{m}=2 f+e_{s}+l_{p}+e_{i}
$$

- Manifold-Shells $\left(s_{m}\right)=$ Remains the same

- Manifold-Rings $\left(r_{m}\right)=$ Double the internal rings

$$
r_{m}=2 r_{i}
$$

- Manifold-Genus $\left(h_{m}\right)=$ Internal ring as it becomes a hole

$$
h_{m}=r_{i}
$$

\subsubsection{Procedure to validate midsurface}

a) Count the topological entities of the midsurface as per the classification suggested : $f, e_{s}, e_{s r}, e_{r r}, e_{r}, e_{i}, v_{s}, v_{r}, v_{i}, s, h, r$ (more details in Section 3.2)

b) Predict the topological entities of the corresponding thin-walled solid using the equations $(9,10,11,12$, and 13$)$.

a. Predicted solid-faces: $f_{m}=2 f+e_{s}+l_{p}+e_{i}$

b. Predicted solid-edges: $e_{m}=$ $2\left(e_{s}+e_{s r}+e_{r r}+e_{i}\right)+\sum n_{r} e_{r}+v_{s}+v_{i}$

c. Predicted solid-vertices: $v_{m}=2 v_{s}+\sum n_{r} v_{r}$ $+2 v_{i}$

d. Predicted solid-shells-holes: $s_{m}=s=1$, $h_{m}=r_{i}=0, r_{m}=2 r_{i}=0$

e. Non-manifold equation left side: $\chi_{n m l}=v-$ $e+f$

\begin{tabular}{|c|c|c|c|c|c|c|c|c|c|c|c|c|c|c|}
\hline Midsurface & $f$ & $l_{p}$ & $e_{S}$ & $e_{s r}$ & $e_{r}$ & $e_{r r}$ & $e_{i}$ & $v_{S}$ & $v_{r}$ & $v_{i}$ & $f_{m}$ & $e_{m}$ & $v_{m}$ & $\chi_{m *}$ \\
\hline & 1 & 0 & 4 & 0 & 0 & 0 & 0 & 4 & 0 & 0 & 6 & 12 & 8 & 2 \\
\hline & 2 & 2 & 2 & 4 & 1 & 0 & 0 & 4 & 2 & 0 & 8 & 18 & 12 & 2 \\
\hline & 3 & 2 & 3 & 6 & 1 & 0 & 0 & 6 & 2 & 0 & 11 & 27 & 18 & 2 \\
\hline
\end{tabular}

f. Non-manifold equation right side: $\chi_{n m r}=$ $s-h+r$

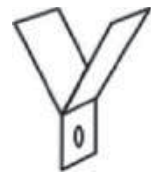
3

23

$6 \quad 1$
$0 \quad 1$

6
2
1230
$20 \quad 2$
3

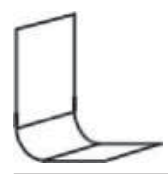

242

Tab. 3: Validation of midsurface. 


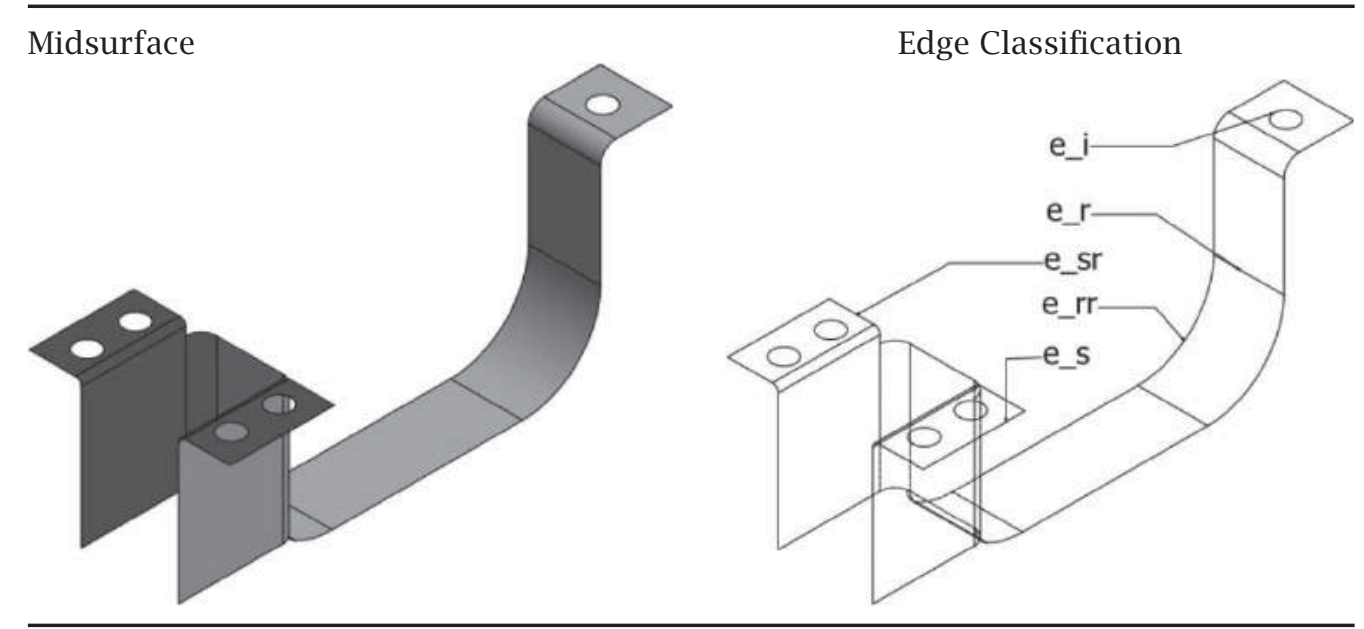

g. Manifold equation left side: $\chi_{m l}=v_{m}-$ $e_{m}+f_{m}$

h. Manifold equation right side: $\chi_{m r}=2\left(s_{m}-\right.$ $\left.h_{m}\right)+r_{m}$

c) Verify that the topological entities of the midsurface satisfy the non-manifold equation (Equation 4$)$, by deducing that the left $\left(\chi_{n m l}\right)$ and right $\left(\chi_{n m r}=\right)$ hand side of the equation match.

d) Verify that the predicted topological entities of the thin-walled solid satisfy the manifold equation (Equation 3), by deducing that the left $\left(\chi_{m l}\right)$ and right $\left(\chi_{m r}\right)$ hand side of the equation match; thus proving that the transformation equations are valid.

\subsubsection{Examples}

Table 3 displays the validation of a midsurface using the proposed dimension-addition-transformation equations. It is evident that the derived formulation works for simple shapes.

Following is the verification for a relativelycomplex practical shape.

- Midsurface entities: $f=15 ; e_{s}=3$;

$e_{s r}=10 ; e_{r}=14 ; e_{r r}=19 ; l_{p}=9 ; e_{i}=5$;

$v_{S}=8 ; v_{r}=24 ; v_{i}=5 ; s=1 ; h=5$;

$r=5$

- Predicted solid-faces: $f_{m}=2 f+e_{s}+l_{p}+e_{i}=$ $2 \times 15+3+9+5=47$

- Predicted solid-edges: $e_{m}=$

$2\left(e_{s}+e_{s r}+e_{r r}+e_{i}\right)+\sum n_{r} e_{r}+v_{s}+v_{i}=$

$2(3+10+19+5)+(2 \times 12+4 \times 2)$

$+8+5=119$

- Predicted solid-vertices: $v_{m}=2\left(v_{s}+v_{i}\right)$ $+\sum n_{r} v_{r}=2 \times(8+5)+2 \times 24=74$

- Predicted solid-shells, holes: $s_{m}=s=1 ; h_{m}=$ $r_{i}=5 ; r_{m}=2 r_{i}=10$
- Non-manifold equation left side: $\chi_{n m l}=v-e+$ $f=32-46+15=1$

- Non-manifold equation right side: $\chi_{n m r}=s-$ $h+r=1-5+5=1$

- Manifold equation left side: $\chi_{m l}=v_{m}-e_{m}+$ $f_{m}=74-119+47=2$

- Manifold equation right side: $\chi_{m r}=2\left(s_{m}-\right.$ $\left.h_{m}\right)+r_{m}=2(1-5)+10=2$

It can be observed that the predicted solid entities validate the manifold equation $\left(\chi_{m l}=\chi_{m r}=2\right)$. Validation can also be performed by comparing the topological entities of the thin-walled solid with the predicted ones.

\section{CONCLUSION}

This paper proposes a new way of topological validation of the midsurface, computed from sheet metal solids. Validation is performed in both the directions, solid-to-midsurface and midsurface-to-solid. The solid-to-midsurface method heavily depends on the quality of the cellular decomposition of the original solid. If the cells formed are not 'clean' or have degeneracies like dangling edges and vertices, the validation results could be unpredictable.

The primary advantage of the proposed method is that it just needs counts of the topological entities, making it quick and robust, in comparison with the geometric methods, which tend to be computationally extensive and approximate.

Another advantage is that changes in the geometry of the shape do not affect the validation criterion, as long as the topology remains the same. So, a plate having planar surfaces or curved ones or having tapered draft angles, all can follow the same topological invariant $\chi_{\text {smm }}$ developed in this method.

The examples show that the newly-derived formulation works not only for the simple shapes but also for a relatively-complex practical part. 


\section{REFERENCES}

[1] Boussuge, F.; Léon, J.-C.; Hahmann, S.; Fine, L.: Extraction of generative processes from b-rep shapes and application to idealization transformations, Computer-Aided Design, 46, 2014, 79-89. http://dx.doi.org/10.1016/j.cad.2013. 08.020 .

[2] Boussuge, F.; Léon, J.-C.; Hahmann, S.; Fine, L.: Idealized models for FEA derived from generative modeling processes based on extrusion primitives, 2014, 127-145 http://dx.doi.org/ 10.1007/978-3-319-02335-9-8.

[3] Briere-Cote, A.: Semantic characterization of differences between cad models through explicit geometric constraint transpositions, Ph.D. thesis, University of Quebec, 2014.

[4] Cao, W.; Chen X.; Gao S.: An approach to automated conversion from design feature model to analysis feature model, Proceedings of the ASME 2011 International Design Engineering Technical Conferences \& Computers and Information in Engineering Conference, 5, 2011, 655-665. http://dx.doi.org/10.1115/DETC 2011-47555.

[5] Chen, G.; Ma, Y. S.; Thimm, G.; Tang, S. H.: Using cellular topology in a unified feature modeling scheme, ComputerAided Design and Applications, 3, 2006, 8998. http://dx.doi.org/10.1080/16864360.2006. 10738445

[6] Chong, C. S.; Kumar, A. S.; Lee, K. H.: Automatic solid decomposition and reduction for non-manifold geometric model generation, Computer-Aided Design, 36, 2004, 1357-1369. http://dx.doi.org/10.1016/j.cad.2004.02.005.

[7] Iyer, N.; Jayanti, S.; Lou, K.; Kalyanaraman, Y.; Ramani, K.: Three-dimensional shape searching: State-of-the-art review and future trends, Computer-Aided Design, 37(5), 2005, 509-530. http://dx.doi.org/10.1016/j.cad.2004.07.002.

[8] Kim, B. C.; Mun, D.: Feature-based simplification of boundary representation models using sequential iterative volume decomposition, 38 , 2014, 97-107. http://dx.doi.org/10.1016j.cag. 2013.10.031.

[9] Krishnamurti R.: A Course on Geometric Modeling: Theory, Programming and Practice, School of Architecture, Carnegie Mellon University, (2002).

[10] Lee, S. H.: Offsetting operations on nonmanifold topological models, Computer-Aided Design, 41(11), 2009, 830-846. http://dx.doi. org/10.1016/j.cad.2009.05.001

[11] Lee, S. H.; Kim, H. S.: Sheet modeling and thickening operations based on non-manifold boundary representations, Proceedings of
DETC01, ASME Design Engineering Technical Conferences, 2001.

[12] Lee, S. H.; Lee, K.: Partial entity structure: A compact non-manifold boundary representation based on partial topological entities, Proc. SMA'01, 2001, 159-170. http://dx.doi.org/10. 1145/376957.376976.

[13] Lipson, H.; Shpitalni, M.: On the Topology of Sheet Metal Parts, Journal of Mechanical Design, 120(1), 1998, 10-16. http://dx.doi.org/ 10.1115/1.2826661.

[14] Lockett, H.; Guenov, M.: Similarity measures for mid-surface quality evaluation, Computer-aided Design, 40(3), 2008, 368-380. http://dx.doi.org/10.1016/j.cad.2007.11.008

[15] Rezayat, M.: Midsurface abstraction from 3D solid models: general theory, applications, Computer-Aided Design, 28, 1996, 905915. http://dx.doi.org/10.1016/0010-4485(96) 00018-8

[16] Sequin, C. H.: Generalized Euler-Poincaré theorem, http://www.cs.berkeley.edu/ sequin/ PAPERS/EulerRel.pdf (Last accessed Feb 2015)

[17] Sheen, D.-P.; Geun Son, T.; Myung, D.-K.; Ryu, C.; Lee, S. H.; Lee, K.; Yeo, T. J.: Solid deflation approach to transform solid into mid-surface, Proceedings of the TMCE, 2010. http://dx.doi.org/10.1016/j.cad.2010.01.003.

[18] Thakur, A.; Banerjee, A. G.; Gupta, S. K.: A survey of CAD model simplification techniques for physics-based simulation applications, Computer-Aided Design, 41, 2009, 65-80. http://dx.doi.org/10.1016/j.cad.2008.11.009

[19] Treeck, C. V.; Romberg, R.; Rank, E.; Für, L.: Simulation based on the product model standard ifc, in: In Proc. 8th IBPSA Conference, (2003). http://dx.doi.org/10.1.1.322.1583

[20] Weiler, K.: Topological structures for geometric modeling, Ph.D. thesis, Rensselaer Polytechnic Institute, 1986.

[21] Woo, Y.: Fast cell-based decomposition and applications to solid modeling, ComputerAided Design, 35, 2003, 969-977. http://dx. doi.org/10.1016/S0010-4485(02)00144-6.

[22] Woo, Y.; Kim, S.-H.: Protrusion recognition from solid model using orthogonal bounding factor, Journal of Mechanical Science and Technology, 28(5), 2014, 1759-1764. http://dx.doi.org/10.1007/s12206-014-0322-0.

[23] Woo, Y.; Sakurai, H.: Recognition of maximal features by volume decomposition, ComputerAided Design, 34, 2002, 195-207. http://dx. doi.org/10.1016/S0010-4485(01)00080-X.

[24] Wu, H.; Shuming, G.: Automatic swept volume decomposition based on sweep directions extraction for hexahedral meshing, 23rd 
International Meshing Roundtable, 82, 2014, 13-148. http://dx.doi.org/10.1016/j.proeng. 2014.10.379.

[25] Yamaguchi, F.: Computer-Aided Geometric Design: A Totally Four-Dimensional Approach, chap. Fundamentals of Topology, Springer-
Verlag New York, Inc., Secaucus, NJ, USA, 2002, p. 358.

[26] Yamaguchi, Y.; Kimura, F.: Non-manifold topology based on coupling entities, IEEE Computer Graphics and Applications, 15, 1995, 42-50. http://dx.doi.org/10.1109/38.364963. 